\title{
Revascularização do miocárdio no octogenário: observação de 16 anos
}

José Carlos R. IGLÉZIAS*, Luís Alberto DALLAN*, Artur LOURENÇÃO JÚNIOR*, José Antônio F. RAMIRES ${ }^{\star}$, Noedir A. G. STOLF* ${ }^{\star}$, Sérgio Almeida de OLIVEIRA*, Geraldo VERGINELLI*, Adib D. JATENE*

RBCCV 44205-246

IGLÉZIAS, J. C. R.; DALLAN, L. A.; LOURENÇĀO JÚNIOR, A.; RAMIRES, J. A. F.; STOLF, N. A. G.; OLIVEIRA, S. A.; VERGINELLI, G.; JATENE, A. D. - Revascularização do miocárdio no octogenário: observação de 16 anos. Rev. Bras. Cir. Cardiovasc., 9 (3): 170-175, 1994.

RESUMO: Com o objetivo de avaliar a evolução do tratamento cirúrgico da doença arterial coronária no paciente octogenário, foram analisados, retrospectivamente, os pacientes com idade maior ou igual a 80 anos submetidos a revascularização do miocárdio no InCor, no período entre janeiro/1978 e agosto/1994. Do total de 79 pacientes, $60(75,94 \%)$ eram do sexo masculino e $19(24,05 \%)$ do sexo feminino. A média de idade foi igual a 82,33 ( 80 a 90 anos). A indicação operatória foi devida ao quadro de angina instável em 56 ( $70,88 \%)$, a angina estável em $22(27,84 \%)$ e $1(1,26 \%)$ paciente foi operado devido à dissecção de placa aterosclerótica quando da realizaçāo de angioplastia. A operaçāo ocorreu em caráter de emergência em $8(10,12 \%)$, em caráter de urgência em $23(29,11 \%)$ e eletivamente em $48(60,75 \%)$ pacientes. A veia safena foi empregada em $69(87,34 \%)$, enquanto a artéria torácica interna pediculada foi utilizada em $10(12,65 \%)$ pacientes. A mortalidade hospitalar global atual é de $6,32 \%$, e vem decrescendo na Instituição. Passou de $13,33 \%{ }^{7}$ em 1990 para $8,5 \%^{8}$ em 1993 e, atualmente, está em $6,32 \%$. As causas de óbito hospitalar foram: encefalopatia anóxica, insuficiência respiratória, hemorragia digestiva é um óbito devido a choque cardiogênico. O tempo médio de seguimento foi de 18,3 (4 a 83) meses. A mortalidade tempo relacionada foi devida a infecção, neoplasia, acidente vascular cerebral, trombose mesentérica, síndrome depressiva e choque cardiogênico. Considerando-se que a população brasileira vem envelhecendo aumentando sua expectativa de vida; que a mortalidade hospitalar para o tratamento cirúrgico da doença arterial coronária no idoso vem decrescendo, apesar do número crescente de operaçōes, concluímos que o tratamento cirúrgico representa boa alternativa ao octogenário portador de doença arterial coronária, pois Ihe permite melhorar a qualidade e expectativa de vida.

DESCRITORES: miocárdio, revascularização, cirurgia, pacientes idosos.

\section{INTRODUÇĀO}

O número de pessoas idosas vem crescendo em nossa sociedade, em termos relativos e absolutos. Na América do Norte este segmento da população cresceu mais do que duas vezes, considerando-se a taxa geral para a população, e irá constituir $20 \%$ ou $25 \%$ do total, na primeira metade do próximo século ${ }^{13}$. Já que a prevalência da doença arterial coronária aumenta com a idade, a demanda para Serviços cardiológicos irá aumentar da mesma maneira ${ }^{3}$. Nos Estados Unidos, um terço dos es- tudos hemodinâmicos é realizado nos pacientes idosos 9, 15. Apesar do declínio na taxa de mortalidade anual, a doença arterial coronária permanece como uma das mais importantes causas de óbito no idoso e está também associada a importante morbidade e limitações ${ }^{14}$.

Quando o tratamento clínico é insuficiente para obter melhora sintomática, a revascularização pode ser cogitada. Entretanto, este procedimento é associado a elevada morbidade e mortalidade e é mais dispendioso que o tratamento medicamentoso 10 .

\footnotetext{
Trabalho realizado no Instituto do Coração do Hospital das Clínicas da Faculdade de Medicina da Universidade de São Paulo. Recebido para publicaçăo em outubro de 1994.

* Do Instituto do Coração do Hospital das Clínicas da Faculdade de Medicina da Universidade de São Paulo. Endereço para correspondência: José Carlos Iglézias. Av. Dr. Eneas Carvalho de Aguiar, 44. Divisăo Cirúrgica. CEP: $05403-000$ Săo Paulo, SP. Brasil.
} 
IGLÉZIAS, J. C. R.; DALLAN, L. A.; LOURENÇÃO JÚNIOR, A.; RAMIRES, J. A. F.; STOLF, N. A. G.; OLIVEIRA, S. A.; VERGINELLI, G.; JATENE, A. D. - Revascularização do miocárdio no octogenário: observação de 16 anos. Rev. Bras. Cir. Cardiovasc., 9 (3): $170-175,1994$.

Escalonando, comparativamente, os custos dos cuidados com saúde, por um lado, com as restrições orçamentárias ou outras limitações de recursos financeiros, por outro lado, podemos concluir que existe uma limitação de recursos frente às necessidades quando se pretende extender os cuidados a toda a população.

Entretanto, uma abordagem baseada na análise do risco-benefício para cada paciente deve ser avaliada.

Para muitos pacientes idosos, o estilo de vida ativo e a independência são mais importantes que a longevidade e a terapêutica pode ser um paliativo, ou um solucionador para os sintomas. O tratamento clínico é algumas vezes insuficiente, como resultado das alterações farmacodinâmicas, ou pode ser menos tolerado devido à concomitante terapia medicamentosa para as desordens associadas. A revascularização do miocárdio propicia o alívio sintomático e é bom resultado a longo prazo. A angioplastia é um procedimento não invasivo e pode representar uma alternativa não cirúrgica; entretanto, os idosos geralmente apresentam doença arterial coronária mais avançada, com artérias tortuosas e rígidas, lesões calcificadas e pobre função ventricular 2,3 .

A decisão para a realização da revascularização no paciente idoso deve se basear, sobretudo, no cuidadoso balanço entre os riscos e benefícios. Isto requer avaliação do quadro clínico, anatomia das coronárias e função ventricular, uma vez que estes fatores poderão determinar o sucesso imediato, o risco de complicações maiores e a sobrevida tardia.

São considerados candidatos os pacientes idosos ativos portadores de sintomas refratários ao tratamento medicamentoso, ou que não toleram a terapia medicamentosa e aqueles que aceitam o risco de revascularização do miocárdio desde que o tratamento seja paliativo.

A revascularização convencional do miocárdio é preferível aos outros métodos alternativos, principalmente nos pacientes que necessitam de revascularização completa.

Neste estudo, objetivamos mostrar a evolução da revascularização do miocárdio na Instituição, ressaltando a diminuição nos índices de mortalidade, paralelamente ao aumento do número de procedimentos nos pacientes octogenários.

\section{CASUÍSTICA E MÉTODOS}

Analisamos, retrospectivamente, todos os pacientes com idade maior ou igual a 80 anos, que foram submetidos a revascularização do miocárdio
GRÁFICO 1

DISTRIBUIÇÃO QUANTO AO SEXO

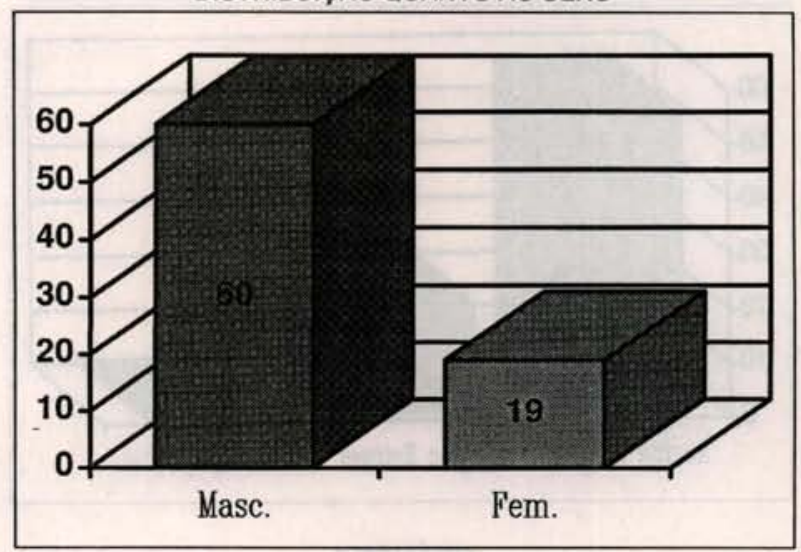

no InCór, no período compreendido entre janeiro/ 1978 e agosto/1994.

Nenhum modelo matemático ou análise estatística foi empregada nesta casuística.

No período analisado, foram revascularizados 79 pacientes, sendo $60(75,94 \%)$ do sexo masculino e $19(24,05 \%)$ do sexo feminino (Gráfico 1$)$. A média de idade foi de 82,33 ( 80 a 90 ) anos (Gráfico 2).

GRÁFICO 2

FAIXA DE IDADE DOS PACIENTES

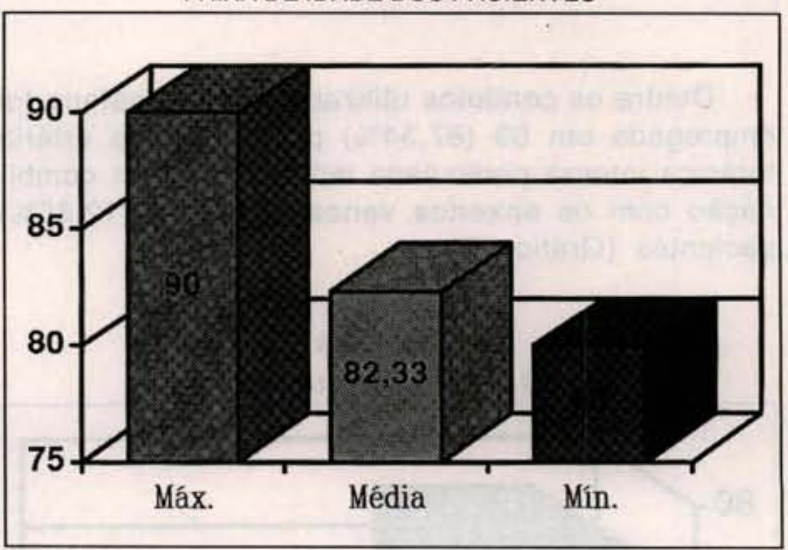

A indicação operatória foi devida ao quadro de angina instável em $56(70,88 \%)$ pacientes, angina estável em outros $22(27,84 \%)$ pacientes e $1(1,26 \%)$ deles foi operado devido à dissecção de placa aterosclerótica durante angioplastia (Gráfico 3).

As operações foram realizadas em caráter de emergência em $8(10,12 \%)$ pacientes, em caráter de urgência em outros $23(29,11 \%)$. Quarenta e oito $(60,75 \%)$ pacientes foram operados eletivamente (Gráfico 4). 
IGLÉZIAS, J. C. R.; DALLAN, L. A.; LOURENÇÃO JÚNIOR, A.; RAMIRES, J. A. F.; STOLF, N. A. G.; OLIVEIRA, S. A.; VERGINELLI, G.; JATENE, A. D. - Revascularização do miocárdio no octogenário: observação de 16 anos. Rev. Bras. Cir. Cardiovasc., 9 (3): 170-175, 1994.

GRÁFICO 3

QUADRO CLINIICO DETERMINANTE DA INDICAÇĀO CIRÚRGICA

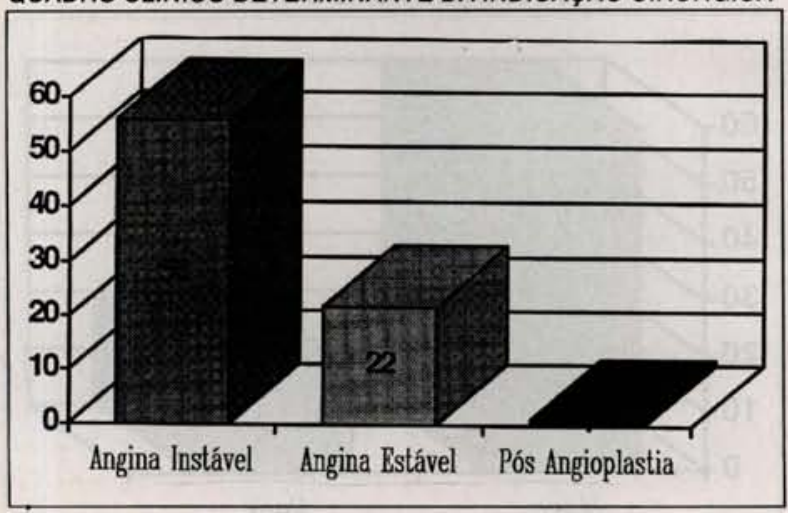

GRÁFICO 4

CARÁTER DA INDICAÇĀO CIRÚRGICA

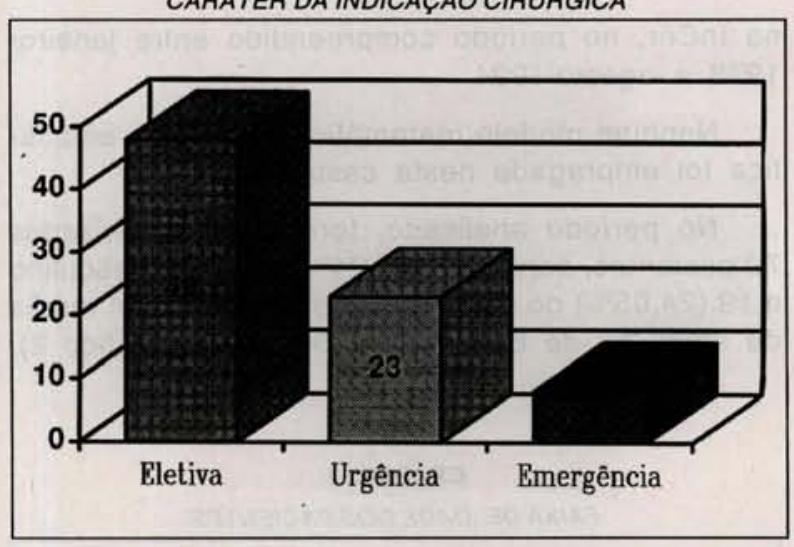

Dentre os condutos utilizados, a veia safena foi empregada em $69(87,34 \%)$ pacientes e a artéria torácica interna pediculada isolada e/ou em combinação com os enxertos venosos, em 10 (12,65\%) pacientes (Gráfico 5 ).

GRÁFICO 5

TIPOS DE ENXERTOS UTILIZADOS

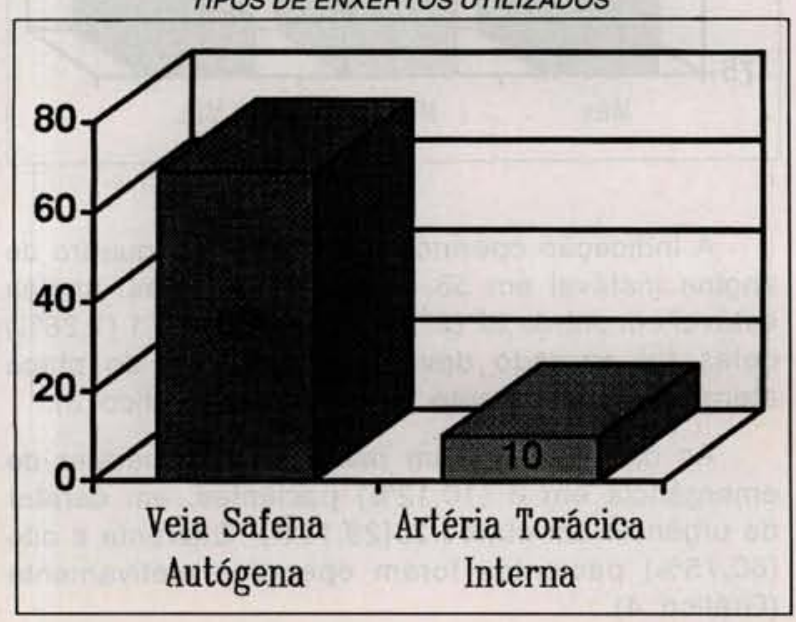

\section{RESULTADOS}

A mortalidade hospitalar vem decrescendo, quando analisamos os períodos estudados, ou seja, passou de $2 / 15(13,33 \%)^{8}$ no período de janeiro/ 78 a julho/1990, para $4 / 47(8,51 \%)^{7}$ no período de janeiro/78 a janeiro/93. Atualmente, está em $6,32 \%$ (Gráfico 6).

\section{GRÁFICO 6}

MORTALIDADE HOSPITALAR NOS PERIOODOS PROGRESSIVOSESTUDADOS

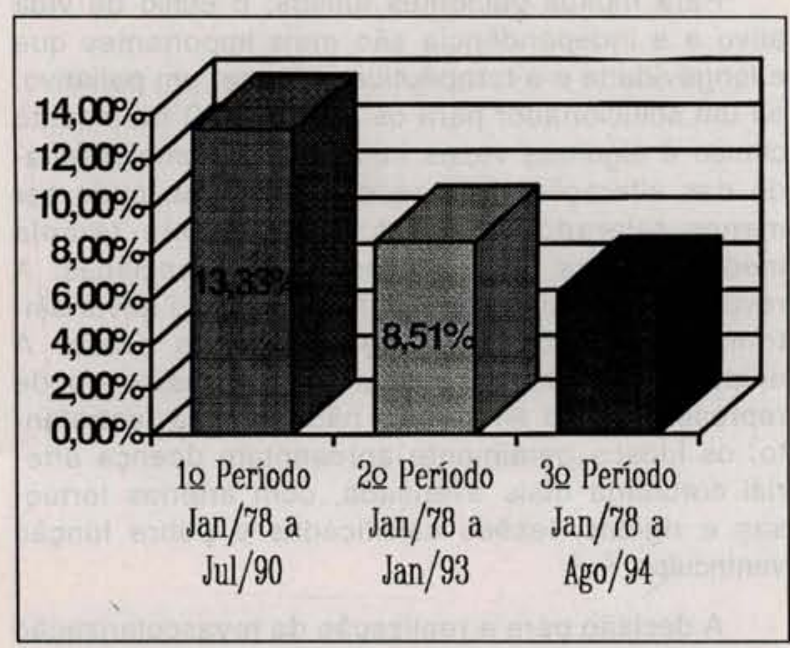

Entre os determinantes do óbito hospitalar, encontramos a encefalopatia anóxica em 2 (2,53\%) pacientes, a insuficiência respiratória em $1(1,26 \%)$ paciente e a hemorragia digestiva em 1 (1,26\%) paciente. Houve uma $1(1,26 \%)$ morte relacionada, que foi devida a choque cardiogênico secundário a infarto agudo do miocárdio (Gráfico 7).

GRÁFICO 7

CAUSAS DETERMINANTES DO ÓBITO HOSPITALAR

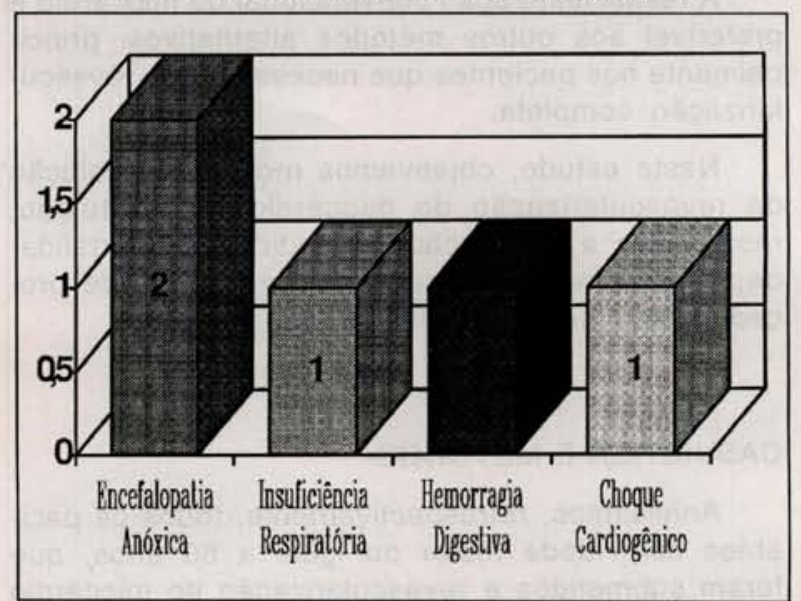


GRÁFICO 8

CAUSAS DETERMINANTES DO ÓBITO TARDIO

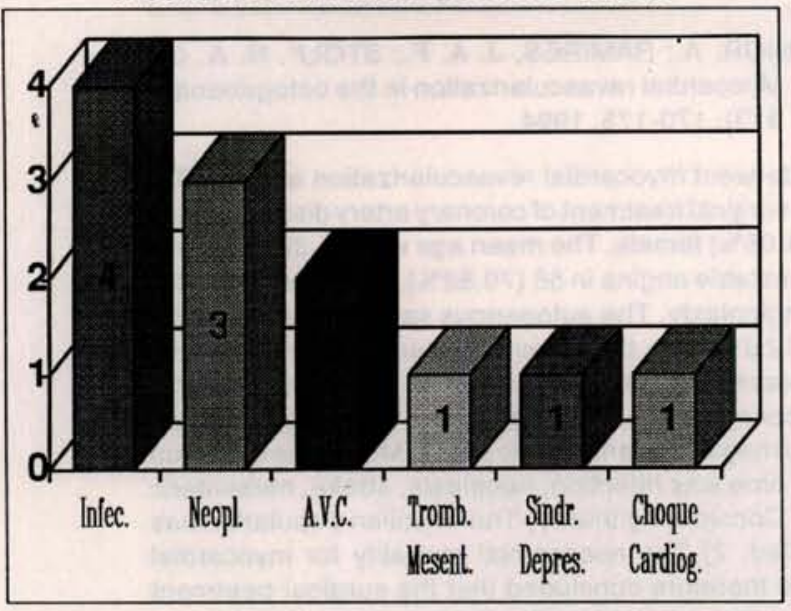

O tempo médio de seguimento foi de 18,3 (4 a 83) meses e houve grande benefício no alívio dos sintomas, tanto em relação a angina, quanto a insuficiência cardíaca.

Entre os fatores determinantes do óbito tardio, encontramos a infecção em $4(5,40 \%)$, as neoplasias em $3(4,05 \%)$, o acidente vascular cerebral em 2 $(2,70 \%)$, a trombose mesentérica em $1(1,35 \%)$, a sindrome depressiva em $1(1,35 \%)$ e $1(1,35 \%)$ óbito relacionado devido a choque cardiogênico secundário a infarto do miocárdio (Gráfico 8 ).

\section{COMENTÁRIOS}

Concordamos com HORVATH et alii ${ }^{6}$, quando afirmam que está plenamente justificada a revascu- larização do miocárdio, uma vez que a sobrevida tardia e a abolição dos sintomas são excelentes nos pacientes idosos, embora a mortalidade hospitalar seja maior, particularmente nos operados em caráter de emergência e/ou urgência.

Nossa casuística está em concordância com os achados de ACINAPURA et alii ${ }^{1}$ e ROSE et alii ${ }^{12}$, quando afirmam que os pacientes idosos apresentam um risco aumentado, cardíaco e não cardíaco, para morbidade e mortalidade após revascularização do miocárdio. A taxa maior de mortalidade, neste grupo etário, parece estar atribuída à falência de outros órgãos que não o coração.

GERSH et alii ${ }^{4,5}$ afirmam que a revascularização do miocárdio parece melhorar a sobrevida e a intensidade dos sintomas, quando comparados com aqueles pacientes submetidos a terapia medicamentosa isolada. Esta observação vem de encontro aos nossos achados.

Dentre os determinantes do óbito hospitalar citado por RABELO et alii ${ }^{11}$, figuram, em ordem decrescente de freqüência, $a$ insuficiência respiratória, seguida pela síndrome do baixo débito cardíaco. Isto não foi observado em nossa casuística.

Partindo da premissa de que a população brasileira vem envelhecendo aumentando sua expectativa de vida e considerando que a mortalidade hospitalar para o tratamento cirúrgico da doença arterial coronária no idoso vem, também, decrescendo, apesar do número crescente de operações, podemos concluir que a revascularização do miocárdio representa boa alternativa ao octogenário portador de doença arterial coronária, pois permite melhor qualidade e expectativa de vida. 
IGLÉZIAS, J. C. R.; DALLAN, L. A.; LOURENÇĀO JÚNIOR, A.; RAMIRES, J. A. F.; STOLF, N. A. G.; OLIVEIRA, S. A.; VERGINELLI, G.; JATENE, A. D. - Revascularização do miocárdio no octogenário: observação de 16 anos. Rev. Bras. Cir. Cardiovasc., 9 (3): $170-175,1994$.

\section{RBCCV $44205-246$}

IGLÉZIAS, J. C. R.; DALLAN, L. A.; LOURENÇÃO JÚNIOR, A.; RAMIRES, J. A. F.; STOLF, N. A. G.; OLIVEIRA, S. A.; VERGINELLI, G.; JATENE, A. D. - Myocardial revascularization in the octogenarian: a 16 years follow-up. Rev. Bras. Cir. Cardiovasc., 9 (3): 170-175, 1994.

ABSTRACT: All patients aged $\geq 80$ years which underwent myocardial revascularization at the InCor Institute were analized in order to establish the evolution of surgical treatment of coronary artery disease. From the total of 79 patients, $60(75.94 \%)$ were male and $19(24.05 \%)$ female. The mean age was $82.33(80$ to 90$)$ years. The symptons presented to indicate surgery was unstable angina in $56(70.88 \%)$, stable angina in 22 $(27.84 \%)$ and $1(1.26 \%)$ patient was operated on after angioplasty. The autogenous saphena vein was the surgical approach in $69(87.34 \%)$ patients while in $10(12.5 \%)$ was the mammary vein. The present total nosocomial mortality rate reaches $6.32 \%$. It has been decreasing at the Institution from $13.33 \%$ in 1990 it came down to $8.5 \%$ in 1993 presently it reaches $6.32 \%$. Nosocomial causes of death were found to be anoxic encephalopathy, respiratory insufficiency, digestive hemorrhage and cardiogenic shock. Mean time follow-up ranged 18.3 ( 4 to 83 ) months. The determinant mortality time was infection, neoplasia, stroke, mesenteric thrombus, depressive syndrome and cardiogenic shock. Considering that: 1) The brazilian population has been getting older with the expectancy of life augmented; 2) The nosocomial mortality for myocardial revascularization in the elderly has been decreasing it is therefore concluded that the surgical treatment represents a good alternative for the octogenarian as it leads to a better quality and expectancy of life.

DESCRIPTORS: myocardial revascularization, surgery, elderly patients.

\section{REFERÊNCIAS BIBLIOGRÁFICAS}

1

ACINAPURA, A. J.; ROSE, D. M.; CUNNINGHAN Jr., J. N.; JACOBOWITZ, I. J.; KRAMER, M. D.; ZISBROD, Z. - Coronary artery bypss in septogenarians: analysis of mortality and morbidity. Circulation, 78 (Supl. 1): 179-184, 1988.

BENTIVOGLIO, L. G.; VAN RADEN, M. J.; KELSEY, S. F.; DETRE, K. M. - Percutaneous transluminal coronary angioplasty (PTCA) in patients with relative contra indications: results from the National Heart, Lung and Blood Institute PTCA Registry. Am. J. Cardiol., 53: 82c-88c, 1984.

CHAITMAN, B. R.; BOURASSA, M. G.; DAVIS, K.; ROGERS, W. J.; TYRAS, D. M.; BERGER, R.; KENNEDY, J. W.; FISHER, L.; JUDKINS, M. P.; MOCK, M. B.; KILLIP, J. - Angiographic prevalence of high risk coronary artery disease in patients subsets (CASS). Circulation, 64: 360-367, 1981.

GERSH, B. J.; KRONMAL, R. A.; FRYE, R. L. - Coronary revascularization in the elderly patient. Circulation, 67: 483-497, 1983.

5

GERSH, B. J.; KRONMAL, R. A.; SCHAFF, H. V. Comparison of coronary artery bypass surgery and medical therapy in patients 65 years of age or older: a nonrandomized study from the coronary artery surgery study (CASS) registry. N. Engl. J. Med., 313: 217-224, 1985.
6 HORVATH, K. A.; PEIGH, P. S.; COUPER, G. S.; COLLINS, J. J.; COHN, L. H. - Favorable results of coronary artery bypass grafting in patients older than 75 years. J. Thorac. Cardiovasc. Surg., 99: 92-96, 1990.

7 IGLÉZIAS, J. C. R.; DALLAN, L. A. O.; OLIVEIRA, S. F.; RAMIRES, J. A. F.; OLIVEIRA, S. A.; VERGINELLI, G.; JATENE, A. D. - Revascularização do miocárdio em octogenários(15 anos de observaçāo). Arq. Bras. Cardiol., 61 (Supl 2): 198, 1993.

8 IGLÉZIAS, J. C. R.; DALLAN, L. A. O.; OLIVEIRA, S. F.; RAMIRES, J. A F.; OLIVEIRA, S. A.; VERGINELLI, G.; PILEGGI, F. J. C.; JATENE, A. D. Revascularização do miocárdio no paciente octogenário. Rev. Bras. Cir. Cardiovasc., 5: 183186, 1990.

9 KASHYAP, M. - Cardiovascular disease in the elderly current considerations. Am. J. Cardiol., $63: 3 \mathrm{H}-4 \mathrm{H}$, 1989.

10 KELLY, M. E.; TAYLOR, G. J.; MOSES, H. W.; MIBEL, F. L.; DOVE, J. J.; BATCHELDER, J. E.; WELLONS, H. A.; SCHNEIDER, J. A. - Comparative cost of myocardial revascularization: percutaneous transluminal angioplasty and coronary artery bypass surgery. J. Am. Coll. Cardiol., 5: 16-20, 1985. 
IGLÉZIAS, J. C. R.; DALLAN, L. A.; LOURENÇĀO JÚNIOR, A.; RAMIRES, J. A. F.; STOLF, N. A. G.; OLIVEIRA, S. A.; VERGINELLI, G.; JATENE, A. D. - Revascularização do miocárdio no octogenário: observação de 16 anos. Rev. Bras. Cir. Cardiovasc., 9 (3): $170-175,1994$.

11 RABELO, R. C.; BERNARDES, R. C.; REIS FILHO, F. A. R.; RABELO, W.; MARINO, M. A.; MARINO, R. L. - Revascularização do miocárdio no idoso. Arq. Bras. Cardiol., 61 (Supl 2): 182, 1993. Analysis of morbidity and mortality in patients 70 years of age and over undergoing isolated coronary artery bypass surgery. Am. Heart J., 110: 341-346, 1985.
13 SATLER, L. F.; GREEN, C. E.; WALLACE, R. C.; RACKLEY, C. E. - Coronary artery disease in the elderly. Am. J. Cardiol., 63: 245-248, 1989.

14 SOTHERLAND, J. E.; PERSKY, V. W.; BRODY, J. A. - Propertionate mortality trends: 1950 through 1986. JAMA, 264: 3178-3184, 1990.

15 STASON, W. B.; SANDERS, C. A.; SMITH, H. C. Cardiovascular care of the elderly: economic considerations. J. Am. Coll. Cardiol., 10: 18A-21A, 1987. 